\title{
Surgical Correction of a Pseudoaneurysm in Common Femoral artery and Arteriovenous fistula between Common Femoral artery and vein, Secondary to a stab injury - Experience in $\mathrm{CMCH}$.
}

\author{
Mohammad Fazle Maruf ${ }^{1}$, Nazmul Hossain ${ }^{1}$, Muhammad Abdul Quaium Chowdhury ${ }^{1}$, Tahmina \\ Akter $^{1}$, Sattyajit Dhar ${ }^{2}$, Mamunur Rahman ${ }^{2}$,Subir Barua ${ }^{2}$, Shuvashish Mazumder ${ }^{2}$ \\ ${ }^{1}$ Department of Cardiothoracic Surgery, Chittagong Medical College, Chittagong, ${ }_{2}$ Department of \\ Cardiac Anesthesia, Chittagong Medical College, Chittagong
}

\begin{abstract}
Keywords:
Arterio-venous

A 23 yrs old young man visited us with the complaints of a pulsatile mass in right groin and a thrill over the medial side of the right thigh which radiate to the hypogastric region for last 3 months following a stab injury on the lateral aspect of right upper thigh. He was diagnosed to have a fistula;

Pseudoaneurysm; Femoral artery;

Femoral vein;

Epidural pseudoaneurysm in common femoral artery and an arteriovenous fistula between common femoral artery and common femoral vein in same region by vascular duplex scanning and CT angiogram. We excised the aneurysm and repaired the arteriovenous fistula tract under epidural anesthesia. Finally we repaired the severed right Common femoral artery and common femoral vein. Postoperative course was uneventful.
\end{abstract}

Abstract

anesthesia.

(Cardiovasc. j. 2016; 8(2): 149-154)

\section{Introduction:}

Trauma is the $3^{\text {rd }}$ leading cause of death among the general population. Vascular injuries represent $3 \%$ of everyday traumas with a high morbidity. Pseudoaneurysms in extremities develop after penetrating trauma in $60 \%$ of arterial injuries. ${ }^{1}$ Stab injury is not uncommon in civil society. It causes sharp penetrating injury in blood vessels. Most of time where it is treated with surface closure and pressure bandage for hemostasis without vessel repair, later it may present as arteriovenous fistula (AVF) or pseudoaneurysm.

Arteriovenous fistulas (AVFs) are abnormal connections between the arterial and venous system that bypass the normal capillary beds. ${ }^{2} \mathrm{~A}$ pseudoaneurysm refers to a defect in an arterial wall which communicates arterial blood with the adjacent extra-luminal space. Blood extravasates out of the artery, but is contained by surrounding tissue and compressed thrombus which form a cavity or sac. The association of pseudoaneurysm and AVF is not uncommon. ${ }^{2,3}$

In 1757, William Hunter ${ }^{4}$ described for the first time, the clinical syndrome of AVF and stated that the disease traumatic AVF arose as a result of transfixation, by a cutting instrument, of an artery and a vein lying closer together, the apertures of these vessels subsequently becoming fused, thus permitting a mutual flow of blood between them. It may present with pulsatile mass, thrill, bruit in affected parts and haemodynamically significant lesions may cause dyspnoea, especially on exercise, lethargy, venous hypertension, varicosities, pain and limb swelling.

Vascular injuries may be potentially lethal and can cause death at the scene. One of the most fascinating and misdiagnosed complications of vascular injuries is the AVF and pseudoaneurysm. They are usually secondary to penetrating trauma and occasionally may be diagnosed later even many years after the injury. ${ }^{5}$

Address of Correspondence: Dr. Mohammad Fazle Maruf, Department of Cardiovascular \& Thoracic Surgery, Chittagong Medical College, Chittagong, Bangladesh. E-mail: mfmaruf@yahoo.com 
We report our results of the surgical management of pseudoaneurysm in Common femoral artery (CFA) and AVF between CFA and common femoral vein (CFV) in Chittagong Medical College Hospital where the vascular surgery facilities were not available before.

\section{Case Study}

A young man of 23 yrs came to us from Ramu, Cox's Bazar with complaints of pulsatile swelling \& pain in right groin, difficulty in walking and swelling of right lower limb for 3 months. He had the history of multiple stab injury 3 months ago. He was stabbed in lateral side of right upper thigh. He was in shock and admitted in a local hospital for treatment. He was managed conservatively. After recovery he was discharged from the hospital. But later he observed a tender pulsatile swelling in his right groin which was gradually increasing in size. He had some difficulties in walking and movement of right lower limb. He also felt gradually increasing thrill in the groin of the affected side. There was some sort of soft tissue infection at the site of penetrating injury, which later apparently healed following regular dressing and antibiotic treatment.

When the patient visited us, we found a pulsatile tensed cystic mass in right groin just lateral to the femoral arterial pulse. Another thrill was present just medial to the femoral artery over femoral vein and it was radiating upwards to the lower abdomen. His all peripheral pulses were present but smaller in volume in right leg in comparison to opposite leg.

A continuous machinery bruit was heard at the medial side of common femoral artery over femoral vein radiating upwards to the lower abdomen. He was normotensive. Chest radiograph showed normal cardiac shadow. Duplex study revealed a cystic mass over and lateral to the Common femoral artery which was pulsatile. In color Doppler "to and fro" blood flow movement present in the cystic mass. A communication (fistula) between CFA and CFV also noticed. CT Angiogram confirmed that the cystic mass was measured 55 $\mathrm{mm} \times 40 \mathrm{~mm} \times 35 \mathrm{~mm}$ and was just proximal to the bifurcation of profunda and SFA. No evidence of arterial dissection was present. Peripheral angiogram showed the site and extension of the AV fistula.

Elective surgery was planned and performed to correct the lesion. Epidural regional anesthesia was given. Patient was in supine position. A longitudinal incision was given over the femoral artery site. Exposure of the external iliac, common

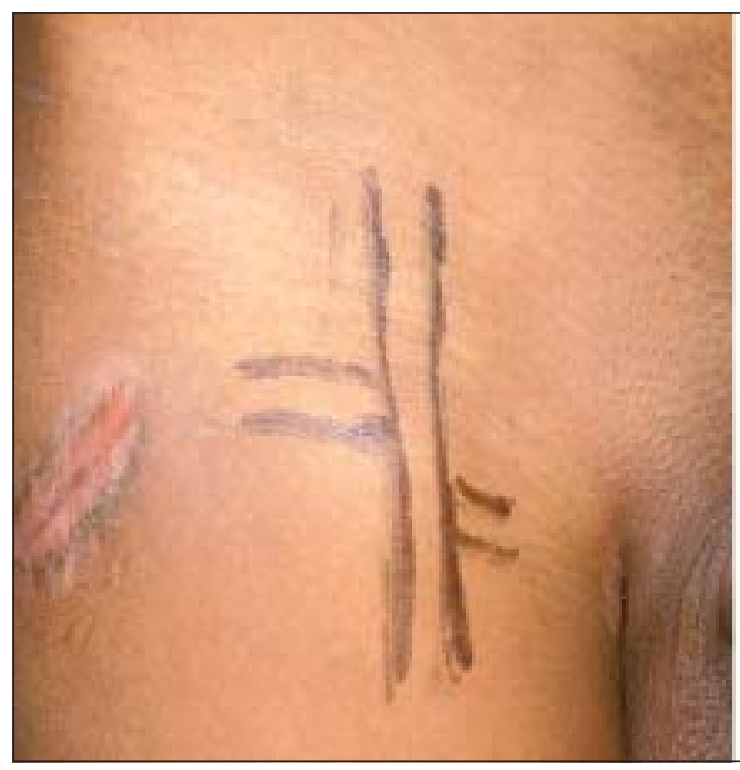

(a)

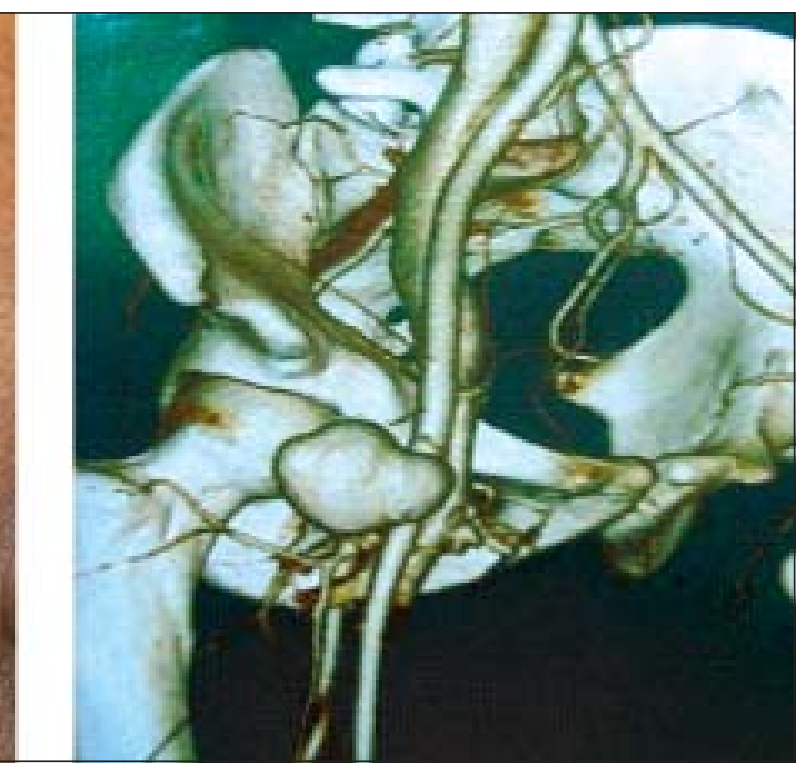

(b)

Fig-1(a): Site of stab injury and pseudoaneurysm in CFA, (b) CT angiogram showing pseudoaneurysm in CFA and $A V F$ between CFA and CFV. 


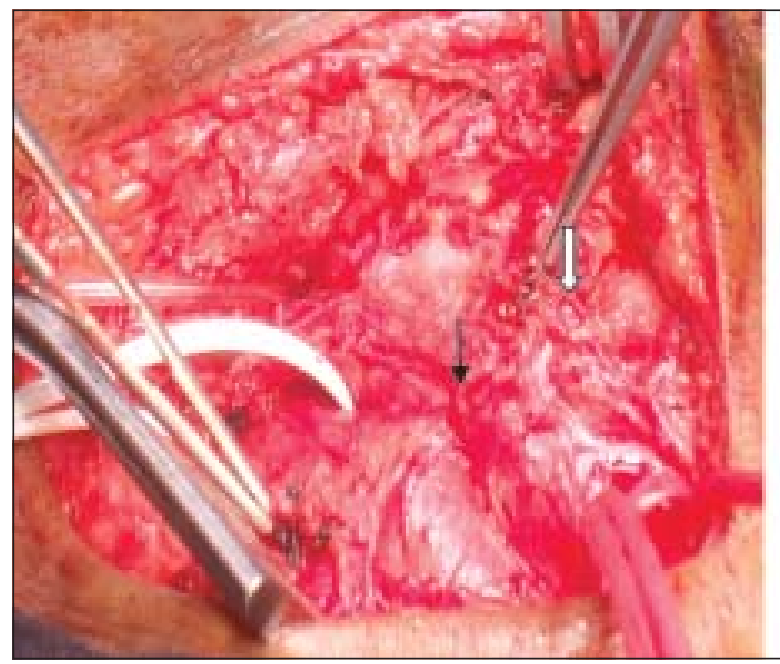

(a)

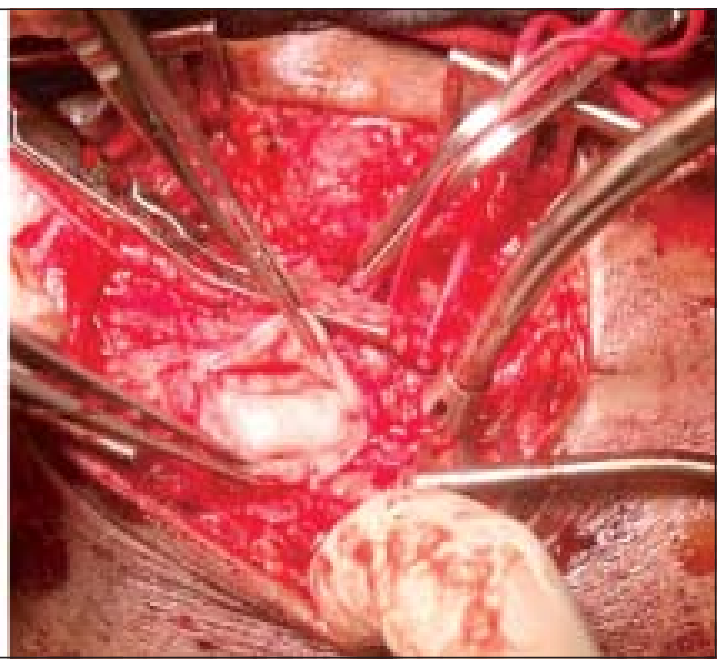

(b)

Fig-2(a): Black arrow-A-V fistula, white arrow pseudoaneurysm (b) Partially excised pseudoaneurysm of CFA.

femoral and superficial femoral arteries was done. Profunda femoris artery could not be visualized. Corresponding veins were also exposed. Proximal control of the external iliac and distal control of the superficial femoral artery with silastic tape was taken. Profunda femoris artery could not be exposed as it was obscured by AV fistula. Site of the arteriovenous fistula was identified. Tape control of the vein just proximal and distal to the fistula was taken. Systemic heparinization was done with 10000 IU of unfractionated heparin IV. All arteries were clamped. Then fistula tract was resected. Arterial and venous walls were repaired with 6-0 prolene. No graft was needed. After that Profunda femoris artery was visualized and control was taken and clamped. Pseudoaneurysm sack was exposed and freed from surroundings. Two large vascular clamps were applied between the femoral arteries and the aneurusmal sac. The sac was resected between the clamps. In order to avoid increasing risk of major hemorrhage or nerve injury, we did not excise the aneurysmal pouch completely. We limited the resection by preserving the adjacent tissues. Pseudoaneurysm sac was opened and marsupialization was done. Embolectomy of CFA, SFA \& deep femoral artery

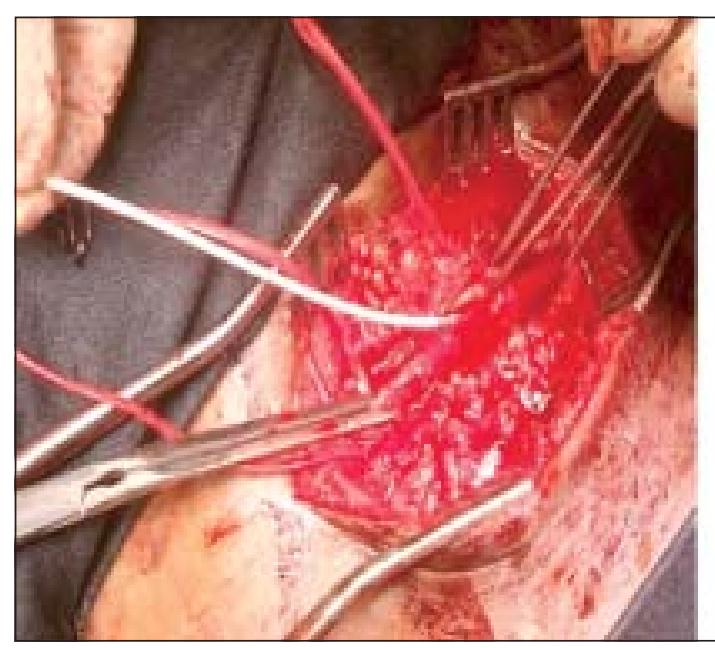

(a)

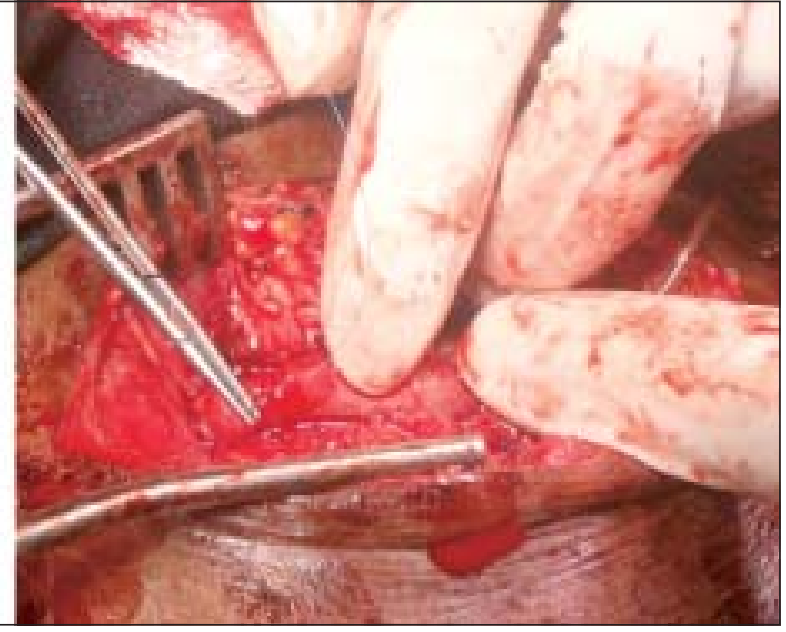

(b)

Fig-3(a): Fogarty embolectomy balloon catheter in SFA, (b) CFA after repair. 
were done with Fogarty embolectomy balloon catheter to confirm adequate antegrade and retrograde flow. CFA was repaired with $6 / 0$ prolene.

Wound was closed after proper hemostasis keeping a drain tube in situ. Distal pulses were intact. The post operative period was uneventful. As the pressure effect of pseudoaneurysm on femoral nerve relieved his pain and difficulties during walk disappeared. He was discharged from hospital on $9^{\text {th }}$ POD. The patient made a good recovery and has been followed up for months. Post operative duplex vascular scan showed normal blood flow in CFA, SFA \& Profunda femoris artery. He is still under our follow-up.

\section{Discussion:}

Post-traumatic AVF is an unusual disease with a wide variability of presentations. AVFs following sharp penetrating injury vary in size. It may be asymptomatic for several months or years. If they persist, a well-developed fistula tract can increase in size leading to significant shunting of blood from the arterial to venous system. The increased venous return can lead to the development of a high output cardiac failure state. If the fistula is large the symptoms may appear at the time of origination. They can be located anywhere in the body, single or multiple, and congenital or acquired (e.g., trauma). Acquired AVFs of the lower extremity are the most commonly occurring AVF. 6

Pseudoaneurysms are the result of traumatic arterial injury. With the increasing utilization of percutaneous arterial interventions, machinery instruments and sharp weapons worldwide, traumatic and iatrogenic arterial injury has become the predominant cause of pseudoaneurysm formation. The highest incidence of iatrogenic pseudoaneurysm formation is observed in the common femoral artery. ${ }^{7}$ There is often a narrow tract stemming from the arterial wall to the pseudoaneurysm sac, termed the 'neck'. A pseudoaneurysm is distinct from a 'true' aneurysm, which results from dilatation of all layers of the arterial wall.

Traumatic AVFs are usually caused by penetrating trauma, accounting for as many as $90 \%$ of cases and blunt trauma is responsible for the other $10 \%$. Gunshot wounds are the most frequent ones, but stab wounds and iatrogenic lesions may also account for traumatic AVFs. ${ }^{8}$
Diagnosis of traumatic AVFs can be made by history of trauma, meticulous physical examination and supported by imaging investigations. Physical examinations with palpation and auscultation may reveal pulsatile mass, thrill, and bruit in affected parts. Distal pulses may be palpable but the AVF can shunt away blood from the extremity and cause symptoms of distal ischemia. Symptoms and sign, from haemodynamically significant lesions include dyspnea, especially on exercise, lethargy, venous hypertension, varicosities, pain, limb swelling and overgrowth. ${ }^{6,9}$ Aneurysmal degeneration of the involved arterial and venous segments may also occur due to structural changes secondary to hemodynamic stress.

Pseudoaneurysms which persist may enlarge and lead to complications related to compression of the adjacent femoral vein, nerve and overlying skin. This can lead to leg swelling, deep vein thrombosis, compressive neuropathy and skin necrosis. Pseudoaneurysms may also expand and eventually rupture. ${ }^{10}$

The differential diagnosis must include true aneurysm, arteriovenous malformation, cyst, abscess and hematoma. One must remember that nonvascular lesions may appear pulsatile due to transmitted pulsation from adjacent arteries.

Diagnosis is usually difficult and greatly dependent on imaging studies. Arteriography was the main exam in the 1960 and 1970s and still plays an important role in diagnosing AVF. Angiography in most cases accurately demonstrates the arteries filling the fistula and the exact point of the arteriovenous communication. Nowadays the computed tomography (CT) angiography, magnetic resonance imaging (MRI) and duplex ultrasonography (DUS) have been playing an important role in the diagnosis of this pathology. ${ }^{11}$

Computed tomographic angiography is a reliable and convenient imaging modality for diagnosing AVF\& pseudoaneurysm. It is a noninvasive modality that could replace conventional arteriography as the initial diagnostic study for arterial injuries after trauma, even when the suspected diagnosis is an AVF and pseudoaneurysm. Duplex ultrasonography (DUS) is the modality of choice for diagnosis of femoral 
pseudoaneurysms and fistulas. ${ }^{12}$ DUS has been reported to have a sensitivity of $94 \%$ and a specificity of $97 \%$ in the detection of femoral pseudoaneurysms. ${ }^{12}$ On DUS, a pseudoaneurysms appears as a hypoechoic sac adjacent to the affected artery, with colour flow observed within it. Thrombus may be identified within part of the sac. The hallmark of diagnosis is the demonstration of a neck communicating between the sac and the affected artery, with a 'to-and-fro' waveform. ${ }^{3}$

Regarding treatment, early recognition and prompt repair may lead to a significant decrease in the number of complications. AVFs do not resolve spontaneously in the majority cases. The aim of the treatment is the closure of the AVF, with preservation of patency of the main vessels.

A proportion of iatrogenic femoral pseudoaneurysms will resolve spontaneously without any form of intervention. An accepted approach is to monitor small $(<3 \mathrm{~cm})$, stable, asymptomatic pseudoaneurysms, as the majority of them will thrombose within 4 weeks. In one large series of small $(<3 \mathrm{~cm})$ pseudoaneurysms, Toursarkissian et al reported a rate of spontaneous thrombosis of $90 \%$ at 60 days of follow up. ${ }^{13}$

Watchful waiting for spontaneous closure with serial ultrasound follow-up and a trial of ultrasound guided compression are appropriate first line treatment options, although less likely to be successful for patients with large AVF with pseudoaneurysm. ${ }^{14}$

Treatment of peripheral arterial aneurysm and AVF includes ultrasound guided compression, ultrasound guided Thrombin Injection, ${ }^{15}$ endovascular intervention ${ }^{16}$ and surgery. ${ }^{17}$ Surgery is associated with a higher initial success rate than other treatments and this benefit is maintained in follow-up. ${ }^{17}$ With the increasing availability of DUS during the 1990s, less invasive treatment options using DUS- guidance gained popularity. Fellmeth et al in 1991 included ultrasound- guided compression (UGC) and percutaneous ultrasound-guided thrombin injection (UGTI) and proposed it as an alternative to surgery. ${ }^{15,16}$ Ultrasound- Guided Thrombin injection (UGTI) of pseudoaneurysms is a technique first described by Kang et al in $1998 .{ }^{18}$ Published study has documented rates of thrombosis between
86-100\%. The most serious complication of UGTI is distal arterial embolisation.

To avoid the morbidity and mortality associated with surgery for traumatic AVFs, the focus has shifted to endovascular techniques. The main advantage of endovascular approach is that the system can be inserted through a remote access site, obviating the need for extensive surgical exposure. Endovascular repair can include the use of covered stents and coil embolization. The first recorded repair of an AVF using an endovascular approach was in 1992 by Parodi ${ }^{16}$ when a covered stent was deployed to treat a traumatic AVF in subclavian vessels.

Endovascular therapy using covered stents and coils are both feasible and safe for treating traumatic AVF. Complications during covered stent and coil placement are due to dissection or rupture and embolization of the devices, which may cause ischemia to the affected parts. ${ }^{16}$

Surgical repair remains the gold standard when an aneurysm is larger than $2.5 \mathrm{~cm}$. The surgical treatment includes open surgical repair, consisting of identification of, the fistula track, aneurysmal sac and its neck under direct vision. ${ }^{7,19}$ Complications include significant hemorrhage from venous hypertension of the arterialized vein and groin infection.

Surgical approach for many of these lesions may be difficult and unfavorable owing to grossly distorted and edematous tissue planes. Arterial and venous repair with the saphenous or prosthetic graft were largely used in the war series and are still used nowadays. Venous ligation, ligationexcision and complex reconstructions may be used. However, late presentation of AVF is prone to significant intraoperative bleeding due to the complex venous anatomy encountered during surgical dissection and repair. Associated morbidities can include limb ischemia, gangrene, limb loss, Venacava thrombosis, pulmonary embolism, venous stasis and uncontrollable bleeding. ${ }^{20}$

In chronic AVF both artery and the vein dilate and elongate in response to the greater blood flow and shear stress, but the vein dilates more and becomes "arterialized". Findings include a pseudoaneurysm, large venous aneurysms, and 
proximal dilatation of the artery or hematoma of the vessel wall.

Due to the reduced venous flow following AVF closure, venous thrombosis can occur especially when venous aneurysms are present, carrying out complications like pulmonary embolism. In this situation systemic anticoagulation is recommended for at least six months after the AVF treatment. Surgical treatment should be performed with vascular capability to prevent complications such as thrombosis, peripheral embolism, rapid expansion or rupture. ${ }^{20}$

\section{Conclusion:}

Currently, vascular injuries are rising with the increase in violence and crime within society. Physical examination is not enough in order to diagnose vascular injuries; therefore, non-invasive and invasive methods must be used in order to diagnose and to exclude possible complications when necessary. This case demonstrates the need for postinjury follow-up to identify missed injuries before they are limb threatening. Despite all the advances in endovascular interventionism, the first treatment option for this pathology is conventional surgery which consists of a resection of the pseudoaneurysm, closure of the AVF tract and reconstruction of artery with or without graft (venous or prosthetic graft).

\section{Conflict of Interest - None.}

\section{Reference}

1. Ucar HI, Oc M, Dooan. Peripheral Vascular Injuries in Civilian Population. Turkiye Klinkleri J Cardiovasc Sci 2006; 18:132-137.

2. Hewitt RL, Smith AD, Drapanas T. Acute traumatic arteriovenous fistulas. J Trauma 1973; 13:901-906.

3. Saad NE, Saad WE, Davies MG, Waldman DL, Fultz PJ, Rubens DJ. Pseudoaneurysms and the role of minimally invasive techniques in their management. Radiographics 2005; 25 Suppl 1: S173-189.

4. William Hunter. The History of an Aneurysm of the Aorta, with some Remarks on Aneurysms in general. Medical Observations and Inquiries 1757; 1: 323-357.

5. Feliciano DV, Bitondo CG, Mattox KL, et al. Civilian trauma in the 1980's. A 1-year experience with 456 vascular and cardiac injuries. Ann Surg 1984; 199:717723.

6. D.S. Sumner. Hemodynamics and pathophysiology of arteriovenous fistulae. In: R.B. Rutherford.Ed. Vascular surgery. Philadelphia,PA: W.B. Saunders, 2000:
7. Ahmad F, Turner SA, Torrie P, Gibson M, Iatrogenic femoral artery pseudoaneurysms-a review of current methods of diagnosis and treatment. Clin Radiol 2008; 63:1310-6.

8. Hughes CW: Arterial repair during the Korean War. Ann Surg 1958; 147:555-561.

9. Creech O, Gantt J, Wren H. Traumatic arteriovenous Fistula at Unusual sites. Ann Surg 1965(June); 161(6), 908-920.

10. De Raet J, Vandekerkhof J, Baeyens I. Ruptured femoral pseudo-aneurysm through the skin: a rare vexing complication following aortobifemoral reconstruction. Acta Chir Belg 2006; 106:420-422.

11. Miller-thomas, MM West, Cohen AM. Diagnosing traumatic arterial injury in the extremities with CT angiography: pearls and pifalls. Radiographics 2005(Oct); 25 (Suppl 1):S133-142.

12. Middleton WD, Dasyam A, Teefey SA. Diagnosis and treatment of iatrogenic femoral artery pseudoaneurysms. Ultrasound Q 2005; 21:3-17.

13. Toursarkissian B, Allen BT, Petrince D et al. Spontaneous closure of selected iatrogenic pseudoaneurysms and arteriovenous fistulae. $J$ Vasc Surg 1997; 25:803-808.

14. Waigand J, Uhlich F, Gross CM, Thalhammer C, Dietz R. Percutaneous treatment of pseudoaneurysms and arteriovenous fistulas after invasive vascular procedures. Catheter Cardiovasc Interv 1999; 47(2):157164.

15. Fellmeth BD, Roberts AC, Bookstein JJ et al. Postangiographic femoral artery injuries: nonsurgical repair with US-guided compression. Radiology 1991; 178:671-675.

16. Yu PT, Rice T.,S. Naheedy,Almodavar H, Mooney DP. Delayed presentation of traumatic infrapopliteal arteriovenous fistula and pseudoaneurysm in a 10 year old boy managed by coil embolization. J Pediatr Surg 2012(Feb);47(2):e7-10.

17. Kuhlencordt PJ, Linsenmeyer U, Rademacher A et al. Large external iliac vein aneurysm in a patient with a post-traumatic femoral arteriovenous fistula. $J$ Vasc Surg 2008; 47:205-208.

18. Kang SS, Labropoulos N, Mansour MA, Baker WH. Percutaneous ultrasound guided thrombin injection: a new method for treating postcatheterization femoral pseudoaneurysms. J Vasc Surg 1998; 27:1032-1038.

19. Feliciano DV, Mattox KL. Traumatic aneurysms. In: Rutherford RB. Eds. Vascular Surgery. Third edition. Philadelphia: W.B. Saunders Company, 1989: 996-1003.

20. Rich NM, Hobson RW, Collins GJ, Traumatic arteriovenous fistulas and false aneurysms:a review of 558 lesions. Surgery 1975; 78:817-828. 